\title{
The Role of OsiriX Based Virtual Endoscopy in Planning Endoscopic Transsphenoidal Surgery for Pituitary Adenoma
}

\author{
Daniel Ilie ROTARIU1', Faiyad ZIYAD' ${ }^{1}$, Alexandru BUDU ${ }^{1}$, Ion POEATA ${ }^{1,2}$ \\ ${ }^{1} \mathrm{~N}$ Oblu Clinical Emergency Hospital, $3^{\text {rd }}$ Neurosurgical Department, lasi, Romania \\ ${ }^{2}$ Gr. T. Popa University of Medicine and Pharmacy, lasi, Romania
}

Portions of this work were presented in poster form at the $11^{\text {th }}$ European Skull Base Society Congress, Paris, France, $26^{\text {th }}-28^{\text {th }}$ June, 2014 and at the $15^{\text {th }}$ Interim Meeting of the World Federation of Neurosurgical Societies, Rome, Italy, $8^{\text {th }}-12^{\text {th }}$ September 2015

\section{ABSTRACT}

AIM: Virtual endoscopy (VE) is the navigation of a 3D model reconstructed from radiological image data. The aim of this paper is to evaluate the role and accuracy of the virtual endoscopy realized using commercially available software, OsiriX, as a method of planning of surgical interventions.

MATERIAL and METHODS: The computed tomographies of 22 patients with pituitary adenomas proposed for endoscopic approach were reconstructed using OsiriX. VE was performed prior to surgery to assess the surgical corridor and particular anatomy. We evaluated the following landmarks: inferior and middle turbinate, sphenoid ostia (SO), choanal arch (Ch), sphenoethmoidal recess (SER), sphenoid septa, sella turcica, carotid prominences and opticocarotid recesses (OCR). The intraoperative endoscopic images were reviewed and compared with the virtual images.

RESULTS: The virtual images had a good resemblance with the actual surgical images. All the structures from the nasal cavity were identified and had a perfect matching except the SO which was identified in 8 cases in VE vs. 12 intraoperative. All the structures from the sphenoid sinus were identified with perfect matching except the ipsilateral carotid prominence (14 in VE vs. 10 intraoperative) and the contralateral carotid prominence (16 in VE vs. 18 intraoperative). The VE could not show the state of the sellar floor and did not offer any information about the sellar content.

CONCLUSION: VE realized in OsiriX represents an affordable alternative to the specially designed systems, offering reliable data and good quality images that are useful for the preoperative planning, but some limitations are present such as inability to obtain additional information in cases where the sphenoid sinus is fully occupied by tumor or not aerated, inability to clearly differentiate between structures at the level of the sella, and impossibility to simulate working instruments.

KEYWORDS: Virtual endoscopy, Pituitary adenoma, Endoscopic endonasal surgery

\section{INTRODUCTION}

$\mathrm{P}$ ituitary adenoma represents the third most common intracranial tumor (15) and the transsphenoidal approach is nowadays the standard approach for most sellar lesions. The endoscopic transsphenoidal approach is the latest innovation in this field, and since its introduction by Jankowski et al. (11) the technique has subsequently been refined and popularized $(4,12)$. The endoscopic technique brings incontestable advantages in terms of illumination, high-resolution, close-up and wide angle view of the structures, the view around the corner, extended maneuverability and decreased trauma of the nasal structures (19), but there are also some disadvantages, caused by the different maneuverability of the surgical instruments, the two dimensional images, and the dif- 
ferent appearance of the anatomical structures $(13,19)$. We, therefore, have proposed to evaluate the role and accuracy of virtual endoscopy (VE) realized in OsiriX as an adjunct in the preoperative planning of the endoscopic endonasal procedures.

\section{MATERIAL and METHODS}

Twenty-two patients with pituitary adenomas, who were operated endoscopically via the transsphenoidal route at the "N Oblu" Clinical Emergency Hospital, lasi, Romania between May 2013 (introduction of the technique) and May 2014, were included in this study. Ethical committee approval and patient consent were obtained. Patient data and characteristics of the lesions are provided in Table I.

The reconstructions were obtained using OsiriX v. 3.8 64bit (Pixmeo ${ }^{\oplus}$ Sarl) for Macintosh, and the system hardware was a regular MacBook Pro $\left(2,3 \mathrm{GHz}\right.$ Intel ${ }^{\circledR}$ Core i7 with a dedicated video card NVIDIA ${ }^{\circledast}$ GeForce GT 650M, 1024MB). The reconstructions of the virtual endoscopic images were obtained using the 3D Endoscopy module and the animations were realized using the Fly Thru module (OsiriX).

The computerized reconstructions were done using computed tomography (CT) images, the scans were obtained using a multi-slice CT scanner, Aquilion (Toshiba, Tokyo, Japan) and the acquisition was realized using a volumetric protocol, from the base of the skull to the vertex. The images used for VE derived from the standard protocol used for patients undergoing transsphenoidal surgery with no additional discomfort, radiation exposure or financial expense.

The surgical interventions were performed by a dedicated team specialized in pituitary surgery. The system used during surgery was a Hopkins II (Karl Storz, Tuttlingen, Germany) with 0 degrees telescope for most of the intervention the other

Table I: Patient Data, Lesion Characteristics

\begin{tabular}{|c|c|}
\hline No. of patients & 22 \\
\hline Female-to-male ratio & $2.14: 1$ \\
\hline Age (yr), median (range) & $52.2(24-78)$ \\
\hline \multicolumn{2}{|l|}{ Pathological features, n (\%) } \\
\hline Nonfunctioning adenoma & $9(40.9 \%)$ \\
\hline PRL cell adenoma & $6(27.3 \%)$ \\
\hline GH cell adenoma & $7(31.8 \%)$ \\
\hline ACTH cell adenoma & $0(0 \%)$ \\
\hline \multicolumn{2}{|l|}{ Imagistic analyze } \\
\hline $\begin{array}{l}\text { Microadenoma-to-macroadenoma- } \\
\text { to-giant adenoma ratio }\end{array}$ & $1: 7.5: 2.5$ \\
\hline Volume $\left(\mathrm{cm}^{3}\right)$, range & $10.48(0.179-34.33)$ \\
\hline Obliteration of sphenoid sinus $\mathrm{n}(\%)$ & $6(27.3 \%)$ \\
\hline
\end{tabular}

GH: Growth hormone, PRL: Prolactin, ACTH: Adrenocorticotropic hormone. angulations $\left(30^{\circ}, 45^{\circ}\right)$ being used just at the end of the surgery for the control of the resection.

The study comprised 2 phases: Phase A in which images were reconstructed preoperatively and were used for the planning of the surgical intervention. In this phase, anatomical variations from the nasal cavity and sphenoid sinus were noted and the surgical route was decided accordingly (Table II). Phase B consisted of revision of the operative videos, and sequences from the videos were compared with the previously virtual reconstructed images. During this process certain landmarks were assessed. For the nasal phase of the procedure the landmarks consisted in identification of the nasal septum (NS), inferior turbinate (IT), middle turbinate (MT), choana (Ch), sphenoethmoidal recess (SER) and sphenoid ostia (SO). For the sphenoid and sellar phase of the procedure the assessed landmarks were: sphenoid septa (SS), sellar floor (SF), opticocarotid recess (OCR), and carotid artery prominences (CA).

The data were analyzed statistically using SPSS for Machintosh, version 22.0 (SPSS, Inc.) Descriptive summaries were reported as mean for continuous variables and as frequency (percentage) for categorical variables. Continuous and categorical data were compared with the t-test and chi-square test, respectively. Correlations were assessed using the Pearson test. A value of $p$ less than 0.05 was considered statistically significant.

\section{RESULTS}

The planning of the surgical intervention using the preoperative virtual endoscopy was possible in all 22 cases (Figure 1A-D).

The virtually reconstructed images had a good resemblance with the intraoperative images (Figure 2A, B). The time for image reconstruction varied between 5 to 30 minutes with a mean of 12 minutes per case. The anatomical structures visualized during the simulation of the approach are listed in Table II.

Nasal Phase: The VE identified the typical landmarks used for orientation during the transsphenoidal approach: inferior and middle turbinate, nasal septum, choanal arch, sphenoid ostia and SER and also some particular anatomical situations that may influence the choice of surgical route or predict the difficulty of the approach such as hypertrophied middle turbinate in $9 \%$ (4 cases), pneumatization of the middle turbinate concha bullosa in $27.3 \%$ (12 cases), deviated nasal septum in $45.5 \%$ ( 10 cases), and septal spur in $27.3 \%$ of the patients (6 cases). In VE, the following anatomical landmarks were identified in $100 \%$ of the cases: inferior and middle turbinate, nasal septum, choanal arch and SER. The landmark with the most variable presence was the sphenoid ostia, which was identified in only 13 of 22 cases (59\%) - assessment was done only for the side of the approach.

The revision of the operative movies revealed a perfect matching for the nasal septum, inferior and middle turbinate, SER and choanal arch with no significant differences between the two methods $(p<0.001 ; p=0.045$ for sphenoid ostia). 
Sphenoid Phase: For the sphenoid sinus we have evaluated the conformation of the sinus and the presence, number and orientation of the sphenoid septa and the landmarks that confine the sellar floor: carotid artery prominences, opticocarotid recess and indentation of the clivus (Figure 3AF).

The VE identified a postsellar conformation of the SS in 12 cases $(54.5 \%)$, a sellar type in 7 cases $(31.3 \%)$, a conchal type in 1 case and a presellar type in 2 cases. The number of sphenoid septa varied between 0 and 4 with a mean of
$1.27 \mathrm{septa} / \mathrm{sinus}$. Multiple septa were identified in 6 patients (2 septa in four patients, 3 septa in one patient and 4 septa in one patient). The preoperative identification of a horizontal septum $(n=2)$ prevented the opening of the sphenoid plane during the preparation of the sellar floor. Comparison of the VE images with the intraoperative ones showed no significantly statistical differences $(p=0.000001)$. The VE missed the presence of a septum in only one case with four septa in the sphenoid sinus. The carotid artery prominences were visualized in 30 out of 44 $(68.1 \%)$ and the same number of OCR. Detailed analyze for the ipsilateral and contralateral carotid artery (depending on

Table II: Anatomical Structures Recognized during the VE and Their Significance

\begin{tabular}{lcc} 
Anatomical structure & No. (\% or range) & Clinical Significance \\
\hline Nasal phase & $10(45.5 \%)$ & Determine side of approach \\
\hline Septal deviation & $6(27.3 \%)$ & \\
\hline Septal spur & $13(29.5 \%)$ & \\
\hline Hypertrophy of the inferior turbinate & $4(9 \%)$ & Open trajectory to sella \\
\hline Hypertrophy of the middle turbinate & $12(27.3 \%)$ & Most constant landmark \\
\hline Concha bullosa & $30(68 \%)$ & \\
\hline Identification of SO & $44(100 \%)$ & Orientation/drilling \\
\hline Identification of the choanal arch & & \\
\hline Sphenoid phase & & \\
\hline Configuration of the SS & $1(4.5 \%)$ & \\
\hline Conchal & $2(9 \%)$ & \\
\hline Presellar & $7(31.8 \%)$ & \\
\hline Sellar & $12(54.5 \%)$ & \\
\hline Postsellar & & \\
\hline Septions & & \\
\hline
\end{tabular}

\section{Septations}

No of septa

Deviated sphenoid septum (not midline centered) Horizontal septum

Onodi cell

Sphenoid landmarks

Carotid prominences

Opticocarotid recess

Indentation of the clivus

\section{Sellar phase}

Sellar floor

$19(86.3 \%)$

Sellar dura

$0(0 \%)$

Pituitary gland

Pituitary adenoma

26 (range 0-4) Orientation within the sinus

$8(30.7 \%)$

$2(8 \%)$

$3(13.6 \%)$

$30(68.1 \%)$

$30(68.1 \%)$

$19(86.4 \%)$ 
the side of the approach) revealed that the ipsilateral carotid artery was identified in VE in 14 cases while on intraoperative images it was identified in only 10 cases, the parametric tests showing a significant statistical difference between the images $(p=0.018)$. The controlateral carotid artery was identified in 16 cases in VE and in 18 cases on operative images, with no significant statistical difference $(p=0.021)$. We have also found that the identification of carotid arteries is influenced by the inferior extension of the tumor $(r=-437, p=0.045)$ and the conformation of the sphenoid sinus $(r=-1, p=0.0001)$ for the sinus concha. The indentation of the clivus was identified in 19 of the 22 patients (86.4\%). The VE could not appreciate the status of the mucosa of the sphenoid sinus.

Sellar Phase: The sellar floor could be identified in $86.3 \%$ of the cases $(n=19)$, but further information was not obtained regarding the status of the sellar floor such as thinned, eroded, or infiltrated. The subtraction of the sellar floor did not provide any information about the subjacent dura or about the content within the sella (Figure 4A-D).
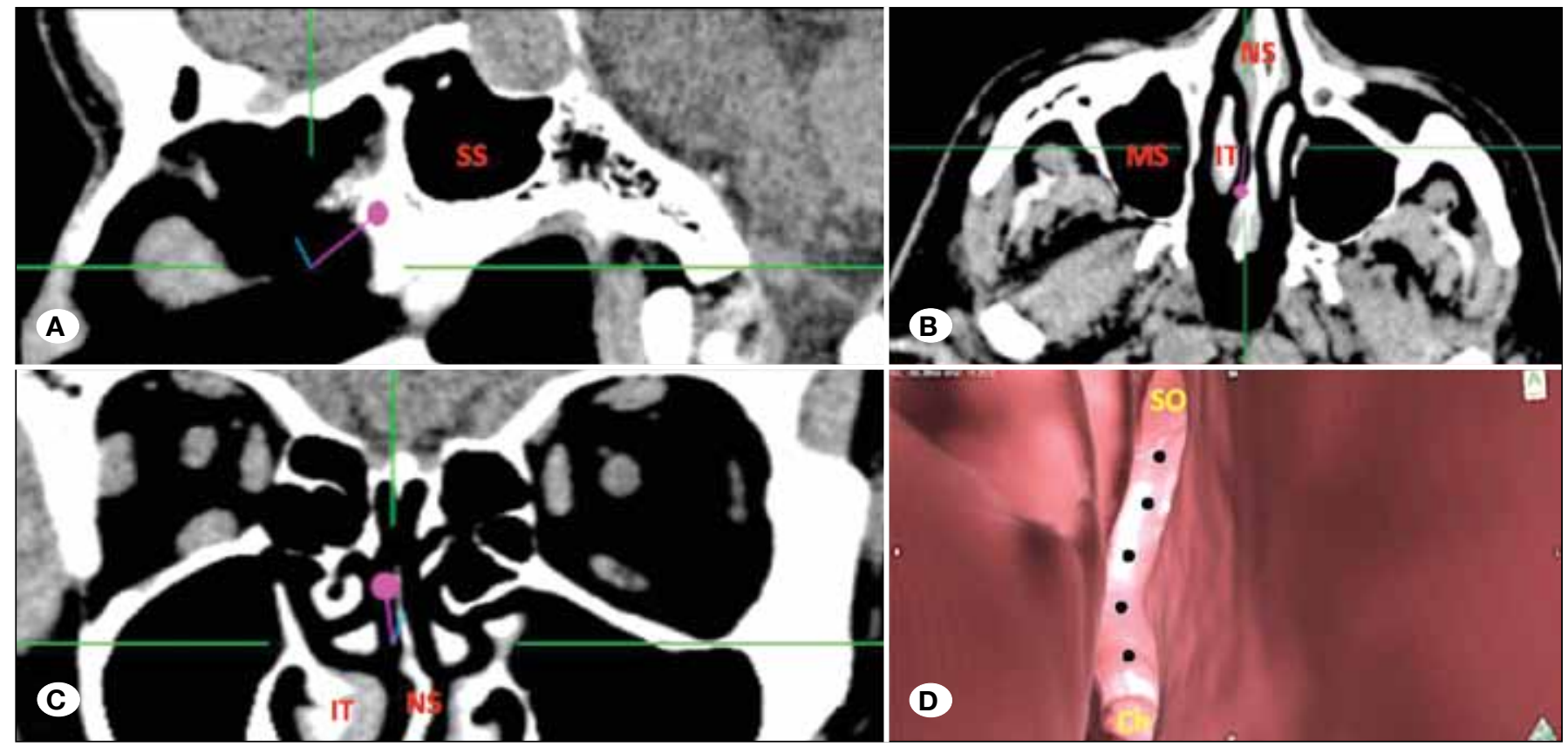

Figure 1: Example of 3D reconstruction of the endoscopic image. A) Position of the camera in the sagittal plane (magenta) with identification of the SS - sphenoid sinus. B) Localization of the camera in axial plane with localization of the MS - maxillary sinus, NS nasal septa and IT - inferior turbinate and C) position in the coronal plane with the camera located in the space bordered by the deviated nasal septum (NS) and inferior turbinate (IT), D) The reconstructed 3D endoscopic image with delimitation of the sphenoethmoidal recess (dotted line) between the choanal arch (Ch) and sphenoid ostia (SO).
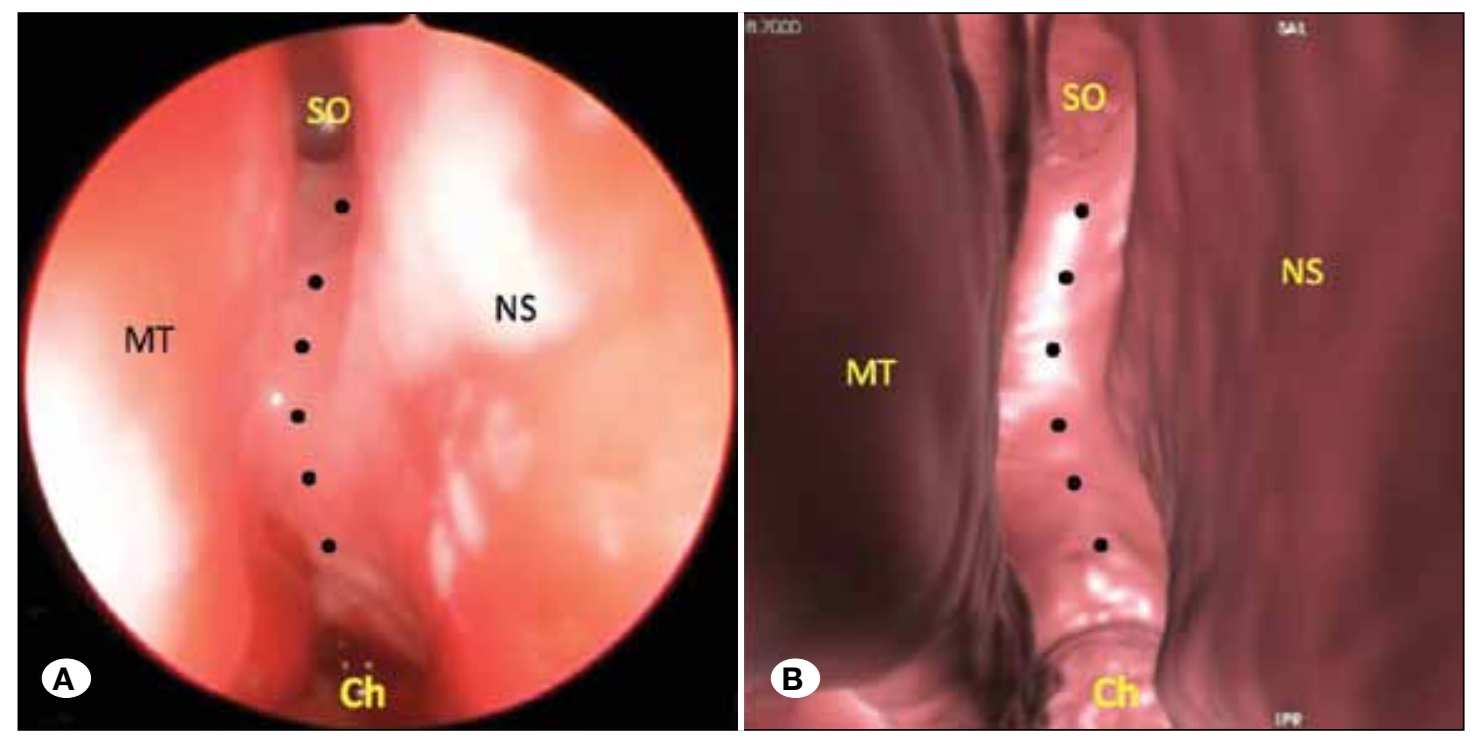

Figure 2:

Identification of anatomical structures at the level of the nasal cavity.

A) intraoperative image and B) virtually reconstructed image, MT: middle turbinate, NS: nasal septum, SO: sphenoid ostia, Ch: choana, dotted line: sphenoethmoidal recess (SER). 


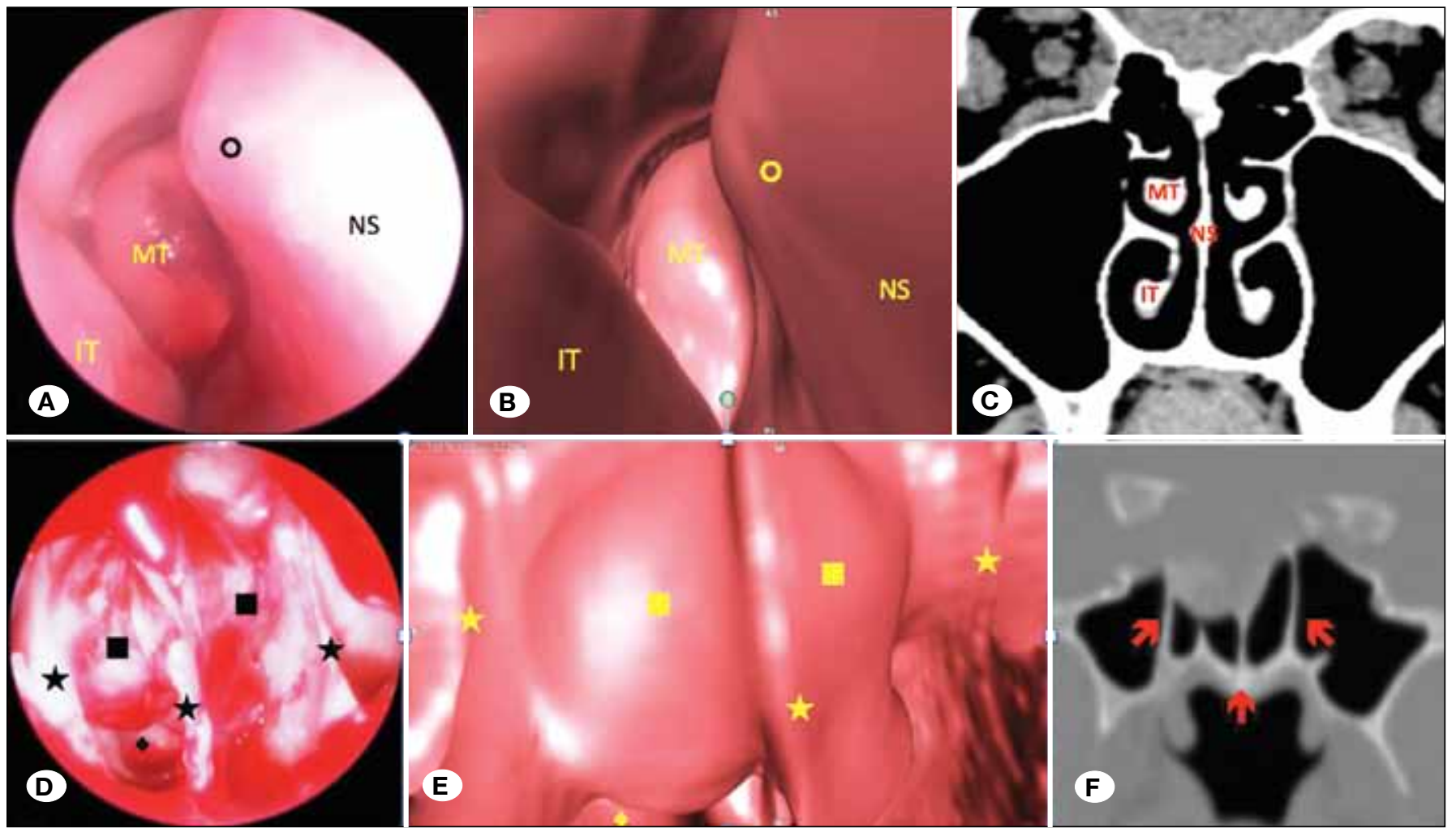

Figure 3: Comparison between intraoperative images (A, D), virtually reconstructed images (B, E) and CT images (C, F). Upper row images from the nasal cavity, MT: Head of the middle turbinate, IT: Inferior turbinate head, NS: Nasal septum with a nasal spur (circle). Lower row images from the sphenoid sinus with identification of multiple septa (stars), enlargement of the sellar floor (square) and identification of the clival indentation (rhomb).
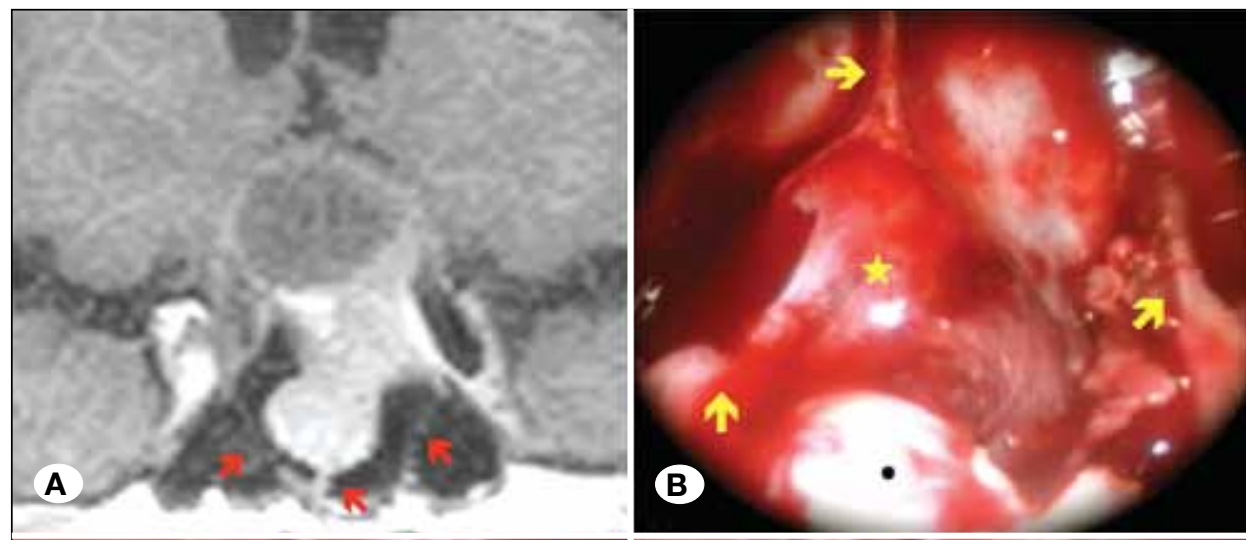

Figure 4: Example of a pituitary adenoma that invaded the sphenoid sinus. A) MRI coronal section shows
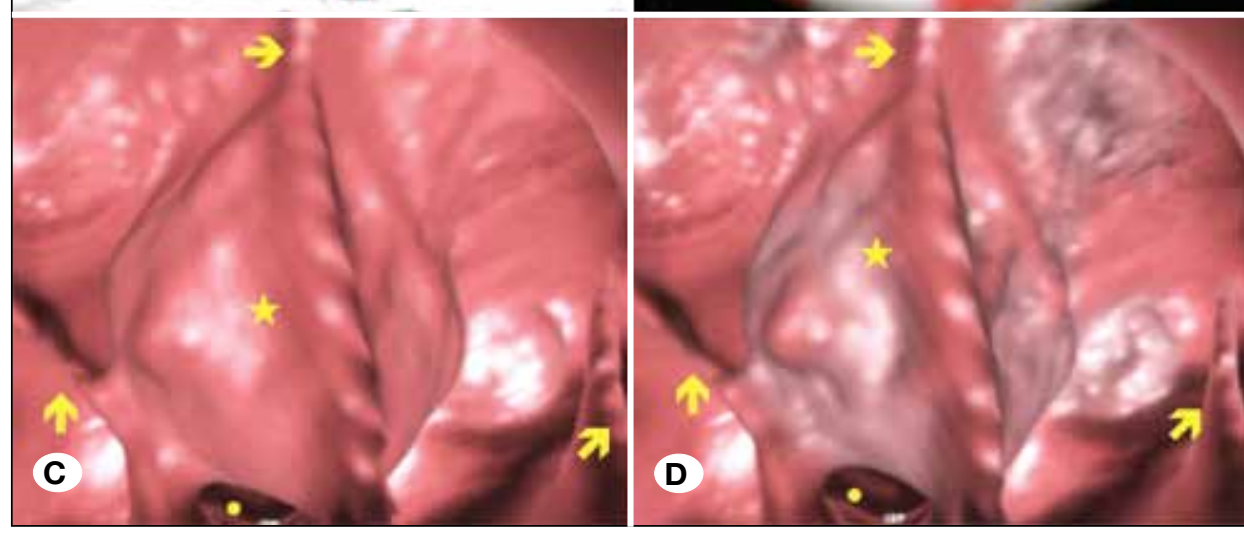
a pituitary lesion with mixt solid and cystic consistency, which extends into the sphenoid sinus that contains multiple septa (arrows).

B) Intraoperative image, showing the inferior extension of the lesion, with complete erosion of the sellar floor and basal dura (star). $\mathbf{C}$ and $\mathbf{D}$ virtually reconstructed images $\mathbf{C}$ native image and $\mathbf{D}$ after maximum subtraction showing the inferior extension of the lesion but with no further information regarding the status of the sellar floor or basal dura. 


\section{DISCUSSION}

The endoscopic transsphenoidal approach has totally replaced the transsphenoidal microsurgical technique used for the treatment of sellar region pathology in some centers in recent years. However, this new technique creates some specific issues for the microscopically based neurosurgeons who are unfamiliar with the 2D magnified, angled and laterally distorted images, different handling of the optic device and surgical instruments, the endoscopic view of the anatomy and working in a narrow channel. Experience, special training and preoperative planning are therefore crucial for the safe use of this technique.

VE represents the navigation of a camera through an air filled cavity that is reconstructed from radiological images. A large range of motion is possible and different angles of view are allowed on these 3D reconstructed images.

Vining was the first to describe the use of VE in 1994, as a post-processing tool for radiologic images (18) and there have been many reports since then on application of VE in other specialties including ORL (14), pneumology $(5,6)$ and urology (1). In neurosurgery, VE was first used by Perneczky in 1998, for the navigation of basal cisterns (2) and then for the exploration of the ventricular system $(3,7)$ and lately for the planning of transsphenoidal surgical interventions (19). All aforementioned pioneers have used dedicated software for the reconstruction of the images.

We propose the commercially available software named OsiriX for the reconstruction of the images and realization of the VE as an alternative to the dedicated software and systems. The images obtained using this method are high quality and have a good resemblance with the intraoperative images. The reliability of the method is sustained by the results obtained after comparison between the virtual and intraoperative images.

VE may be a beneficial training tool for the initiation in endoscopic pituitary surgery as a complementary method to cadaver training and dedicated workshops. The main advantage brought by this method is the possibility of getting familiar with the specific endoscopic anatomy and it also offers the possibility of studying the particular anatomy before surgery. Another advantage is represented by the possibility to become familiar to the specific endoscopic view, with its large, magnified and partially distorted images.

In our clinic we also use VE for the planning of endoscopic surgery and this offers us prospective information about the anatomic variations in the individual patient. VE offers detailed information about the structures from the nasal cavity, aspect and position of the nasal septum (septal deviation, septal spur), aspect of the middle turbinate (hypertrophy of the MT, concha bulosa) thus offering the possibility to appreciate the size of the nasal meatus. The date obtained aid the surgeon in the decision making for the side of the approach, the need for a bilateral approach, or the need for middle turbinate resection.
Furthermore it is essential to know and correctly interpret the sphenoid sinus anatomy in order to realize a safe and individually tailored approach to sellar lesions. The most important landmarks that need to be assessed at the level of sphenoid sinus are the sphenoid septa to have a correct orientation within the sinus and to know their insertion because it is not uncommon for the sphenoid septa to have a lateral attachment in close relation with the carotid artery (9). Other important landmarks are the carotid prominences, medial optic-carotid recess and planum sphenoidale, because these structures are the borders that confine sellar opening in the superior and lateral directions (10) and it is important to know their position in order to avoid opening of the anterior recess and injury of the carotid arteries. In the literature, the cited incidence of carotid injury during endoscopic endonasal skull base surgery ranges from 0.3 to $2 \%$ (8). Talala was the first to describe the use of VE for visualization of the carotid artery inside the sphenoid sinus (17), this technique being important for appreciation of the correct position of the carotid arteries at this level, especially in cases with low inter-carotid distance or carotid procidence. However, with the use of VE reconstructed in OsiriX, limited information is given for the structures located at the level of the sella.

The comparison of the virtual images with their intraoperative counterparts showed a good correlation for the structures located at the level of the nasal cavity and sphenoid sinus confirmed by the significant statistical results. Our method is highly influenced by the degree of pneumatization of the containing structure with negative correlations obtained for the cases of CA identification in patients with invasion of the sphenoid sinus of absence of the pneumatization of the sphenoid sinus.

Compared with the previously described systems $(16,19)$ the reconstructions realized in OsiriX offer limited information about the sellar content, without the possibility to make a clear distinction between the sellar content (tumor, normal pituitary gland, dura mater). The technique is highly dependent by the degree of pneumatization of the sphenoid sinus and does not offer the possibility to simulate instruments, but with all that it is a realistic, viable, and much cheaper alternative.

\section{- CONCLUSION}

VE realized in OsiriX represents an affordable alternative to the specially designed systems, offering reliable data and good quality images that are useful for the preoperative planning and familiarization with the endoscopic anatomy and aspects, but some limitations are present such as inability to obtain additional information in cases where the sphenoid sinus is fully occupied by tumor or not aerated, inability to clearly define between structures at the level of the sella, and impossibility of simulating working instruments. 


\section{REFERENCES}

1. Assimos DG, Vining DJ: Virtual endoscopy. J Endourol 15(1): 47-51, 2001

2. Boor S, Resch KMD, Perneczky A, Stoeter P: Virtual endoscopy (VE) of the basal cisterns: Its value in planning the neurosurgical approach. Minim Invasive Neurosurg 41:177182, 1998

3. Burtscher J, Dessl A, Bale R, Eisner W, Auer A, Twerdy K, Felber S: Virtual endoscopy for planning endoscopic third ventriculostomy procedures. Pediatr Neurosurg 32:77-82, 2000

4. Cudlip S, Joshi SM: Transsphenoidal surgery. Pituitary 11: 353-360, 2008

5. DeWeverW, BogaertJ, Verschakelen JA: Virtual bronchoscopy: Accuracy and usefulness-an overview. Semin Ultrasound CT MR 26: 364-373, 2005

6. Fleiter T, Merkle ME, Aschoff AJ, Lang G, Stein M, Gorich $\mathrm{J}$, Liewald F, Rilinger N, Sokiranski R: Comparison of realtime virtual and fiberoptic bronchoscopy in patients with bronchial carcinoma: Opportunities and limitations. AJR Am J Roentgenol 169: 1591-1595, 1997

7. Freudenstein D, Bartz D, Skalej M, Duffner F: New virtual system for planning of neuroendoscopic interventions. Comput Aided Surg 6: 77-84, 2001

8. Gardner PA, Tormenti, MJ, Pant H, Fernandez-Miranda JC, Snyderman $\mathrm{CH}$, Horowitz MB: Carotid artery injury during endoscopic endonasal skull base surgery: Incidence and outcomes. Neurosurgery 73(2 Suppl Operative): ons261-269; discussion ons269-270, 2013

9. Hamid O, El Fiky L, Hassan O, Kotb A, El Fiky S: Anatomic variations of the sphenoid sinus and their impact on transsphenoid pituitary surgery. Skull Base 18(1):9-15, 2008

10. Jane JA Jr, Han J, Prevedello DM, Jagannathan J, Dumont AS, Laws ER Jr: Perspectives on endoscopic transsphenoidal surgery. Neurosurg Focus 19(6): 1-10, 2005
11. Jankowski R, Auque J, Simon C, Marchal JC, Hepner $H$, Wayoff M: Endoscopic pituitary tumor surgery. Laryngoscope 102(2): 198-202, 1992

12. Jho HD, Carrau RL: Endoscopy assisted transsphenoidal surgery for pituitary adenoma. Technical note. Acta Neurochir (Wien) 138(12): 1416-1425, 1996

13. Leach P, Abou-Zeid AH, Kearney T, Davis J, Trainer PJ, Gnanalingham KK: Endoscopic transsphenoidal pituitary surgery: Evidence of an operative learning curve. Neurosurgery 67: 1205-1212, 2010

14. Martins MJB, Aguiar CV, Júnior JFN, Abreu JPS, Feijão MX, Jataí IO, Bastos ACG: Preoperative planning using 3D reconstructions and virtual endoscopy for location of the frontal sinus. Int Arch Otorhinolaryngol 15(1):48-53, 2011

15. Ostrom QT, Gittleman H, Farah P, Ondracek A, Chen $Y$, Wolinsky Y, Stroup NE, Kruchko C, Barnholtz-Sloan JS: CBTRUS statistical report: Primary brain and central nervous system tumors diagnosed in the United States in 2006-2010. Neuro Oncol 15 Suppl 2: ii1-56, 2013

16. Rosseau G, Bailes J. del Maestro R, Cabral A, Choudhury, Nusrat M, Comas O, Debergue P, De Luca G, Hovdebo J, Jiang Di, Laroche D, Neubauer A, Pazos V, Thibault F, Di Raddo R: The development of a virtual simulator for training neurosurgeons to perform and perfect endoscopic endonasal transsphenoidal surgery. Neurosurgery 73 Suppl 1:85-93, 2013

17. Talala T, Pirila T, Karhula V, Ilkko E, Suramo I: Preoperative virtual endoscopy and three-dimensional imaging of the surface landmarks of the internal carotid arteries in transsphenoidal pituitary surgery. Acta Otolaryngol 120:783-787, 2000

18. Vining DJ, Shifrin RY, Haponik EF, Liu K, Choplin RH: Virtual bronchoscopy. Radiology 193: 261, 1994

19. Wolfsberger S, Neubauer A, Buhler K, Wegenkittl R, Czech T, Gentzsch S, Bocher-Schwarz HG, Knosp E: Advanced virtual endoscopy for endoscopic transsphenoidal pituitary surgery. Neurosurgery 59: 1001-1010, 2006 